\title{
Schwarz Waveform Relaxation Methods for Systems of Semi-Linear Reaction-Diffusion Equations
}

\author{
Stéphane Descombes ${ }^{1}$, Victorita Dolean ${ }^{1}$ and Martin J. Gander ${ }^{2}$ \\ 1 Université de Nice Sophia-Antipolis, Laboratoire J.-A. Dieudonné, UMR CNRS \\ 6621, Parc Valrose, 06018 Nice 02, France, firstname.lastname@unice.fr \\ 2 Université de Genève, Section de Mathématiques, CP 64, 1211 Genève, \\ martin.gander@unige.ch
}

Summary. Schwarz waveform relaxation methods have been studied for a wide range of scalar linear partial differential equations (PDEs) of parabolic and hyperbolic type. They are based on a space-time decomposition of the computational domain and the subdomain iteration uses an overlapping decomposition in space. There are only few convergence studies for non-linear PDEs.

We analyze in this paper the convergence of Schwarz waveform relaxation applied to systems of semi-linear reaction-diffusion equations. We show that the algorithm converges linearly under certain conditions over long time intervals. We illustrate our results, and further possible convergence behavior, with numerical experiments.

\section{Introduction}

Schwarz waveform relaxation methods are domain decomposition methods for evolution problems, which were invented independently in $[1,6]$, and [7], where the latter paper only appeared several years later in print. These methods use a domain decomposition in space, and a subdomain iteration in space-time to converge to the underlying time dependent solution, see Figure 1 for an illustration. Schwarz waveform relaxation methods exhibit different convergence behaviors, depending on the underlying PDE and the time interval of the simulation: for the heat equation, convergence is linear over long times, see [6], and superlinear over short times, see [7]. For the wave equation, convergence is obtained in a finite number of steps for bounded time intervals, see [4], where also an optimized variant is described, which was first proposed in [3], both for hyperbolic and parabolic problems.

The analysis of Schwarz waveform relaxation methods for nonlinear problems is significantly more difficult: for scalar semilinear reaction diffusion problems, see [2], and for scalar convection dominated nonlinear conservation laws, see [5]. The purpose of our paper is to present a first convergence analysis for 
systems of nonlinear PDEs, for the model problem of semilinear reaction diffusion equations.

\section{Systems of Semi-linear Reaction Diffusion Equations}

To simplify the presentation, we show our results for a system of two equations in one spatial dimension, but the techniques used in the analysis can be generalized to systems with more unknowns, and also to higher dimensions. We consider on a bounded domain $\Omega \subset \mathbb{R}$ the system of semi-linear reaction diffusion equations

$$
\begin{aligned}
\partial_{t} \boldsymbol{u}-\Delta \boldsymbol{u}+\boldsymbol{f}(\boldsymbol{u}) & =0 & & \text { in } \Omega \times(0, T), \\
\boldsymbol{u}(x, t) & =\boldsymbol{g}(x, t) & & \text { on } \partial \Omega \times(0, T), \\
\boldsymbol{u}(x, 0) & =\boldsymbol{u}_{0}(x) & & \text { in } \Omega,
\end{aligned}
$$

where $\boldsymbol{u}=\left(u_{1}, u_{2}\right)$ represents the vector of two unknown concentrations to be determined, and $\boldsymbol{f}(\boldsymbol{u})=\left(f_{1}\left(u_{1}, u_{2}\right), f_{2}\left(u_{1}, u_{2}\right)\right)$. A well posedness result for such systems of semi-linear reaction diffusion equations can be found in [8], see Corollary 3.3.5, page 56 .

Our analysis of the Schwarz waveform relaxation algorithm is based on comparison principles. Such principles have been studied in various contexts for system (1), see for example [10], [11], and they often require quite elaborate proofs for the generality employed. We state here precisely the results we need.

Lemma 1. Let $\boldsymbol{u}=\left(u_{j}\right)_{1 \leq j \leq 2} \in C^{2,1}(\Omega \times[0, \infty))^{2}$ be a function for which each component satisfies the inequality

$$
\begin{aligned}
& \partial_{t} u_{i}-\Delta u_{i}+a_{i 1}(x, t) u_{1}+a_{i 2}(x, t) u_{2}>0 \quad \text { in } \Omega \times(0, \infty), \\
& u_{i}(x, t)>0 \quad \text { on } \partial \Omega \times(0, \infty) \text {, } \\
& u_{i}(x, 0)>0 \text { in } \Omega \text {. }
\end{aligned}
$$

If $a_{i j}(x, t) \leq 0$ for $i \neq j$ and all $(x, t) \in \Omega \times(0, \infty)$, then $u_{i}(x, t)>0$ for all $(x, t) \in \Omega \times(0, \infty)$.

The proof of this theorem by contradiction is a straightforward extension of the result in the scalar case, see [2]. The strict inequalities in Lemma 1 can however be relaxed, as we show next.

Lemma 2. Under the same assumptions as in Lemma 1, if

$$
\begin{array}{rlrl}
\partial_{t} u_{i}-\Delta u_{i}+a_{i 1}(x, t) u_{1}+a_{i 2}(x, t) u_{2} & \geq 0 & & \text { in } \Omega \times(0, \infty), \\
u_{i}(x, t) \geq 0 & & \text { on } \partial \Omega \times(0, \infty), \\
u_{i}(x, 0) \geq 0 & & \text { in } \Omega,
\end{array}
$$

then $u_{i}(x, t) \geq 0$ for all $(x, t) \in \Omega \times(0, \infty)$. 
Proof. By performing the change of variables $\tilde{u}_{i}(x, t):=e^{C t} u_{i}(x, t)$, where $C$ is a constant to be chosen, the first inequality of (3) can be rewritten as

$$
\partial_{t} \tilde{u}_{i}-\Delta \tilde{u}_{i}-C \tilde{u}_{i}+a_{i 1} \tilde{u}_{1}+a_{i 2} \tilde{u}_{2} \geq 0 .
$$

Now let $\hat{u}=\tilde{u}+\varepsilon$. If we rewrite (4) in terms of $\hat{u}$, and choose the constant $C$ such that $C<a_{i 1}+a_{i 2}$, we can apply Lemma 1, and taking the limit for $\varepsilon \rightarrow 0$ shows that $u_{i}(x, t) \geq 0$.

\section{Schwarz Waveform Relaxation Algorithm}

We consider the semi-linear reaction diffusion system (1) in the domain $\Omega=$ $(0, L)$. We decompose the domain into two overlapping subdomains $\Omega_{1}=$ $(0, \beta L)$ and $\Omega_{2}=(\alpha L, L), \alpha<\beta$, as shown in Figure 1 . We denote by $\boldsymbol{g}_{\mathbf{1}}(t):=$

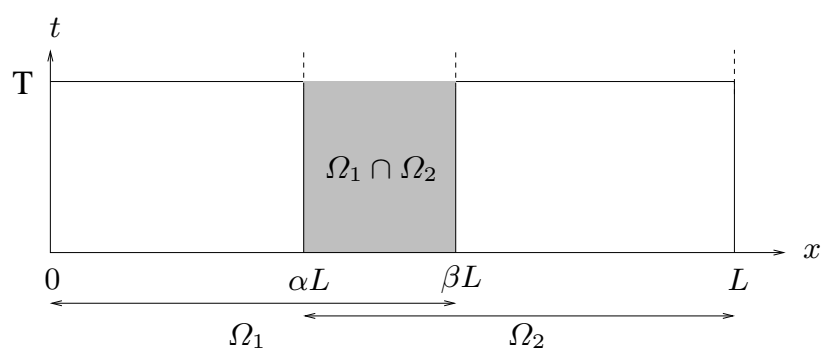

Fig. 1. Space-time domain decomposition.

$\boldsymbol{g}(0, t)$ and by $\boldsymbol{g}_{\mathbf{2}}(t):=\boldsymbol{g}(L, t)$. The classical Schwarz waveform relaxation algorithm constructs at each iteration $n$ approximate solutions $\boldsymbol{v}^{n}, \boldsymbol{w}^{n}$ on subdomains $\Omega_{i}, i=1,2$, by solving the equations

$$
\begin{aligned}
\partial_{t} \boldsymbol{v}^{n+1}-\Delta \boldsymbol{v}^{n+1}+\boldsymbol{f}\left(\boldsymbol{v}^{n+1}\right) & =0 & & \text { in } \Omega_{1} \times(0, T), \\
\boldsymbol{v}^{n+1}(0, t) & =\boldsymbol{g}_{1}(t) & & \text { on }(0, T), \\
\boldsymbol{v}^{n+1}(\beta L, t) & =\boldsymbol{w}^{n}(\beta L, t) & & \text { on }(0, T), \\
\boldsymbol{v}^{n+1}(x, 0) & =\boldsymbol{u}_{0}(x) & & \text { in } \Omega_{1} \\
\partial_{t} \boldsymbol{w}^{n+1}-\Delta \boldsymbol{w}^{n+1}+\boldsymbol{f}\left(\boldsymbol{w}^{n+1}\right) & =0 & & \text { in } \Omega_{2} \times(0, T), \\
\boldsymbol{w}^{n+1}(\alpha L, t) & =\boldsymbol{v}^{n}(\alpha L, t) & & \text { on }(0, T), \\
\boldsymbol{w}^{n+1}(L, t) & =\boldsymbol{g}_{2}(t) & & \text { on }(0, T), \\
\boldsymbol{w}^{n+1}(x, 0) & =\boldsymbol{u}_{0}(x) & & \text { in } \Omega_{2} .
\end{aligned}
$$

In order to analyze the convergence of the Schwarz waveform relaxation algorithm (5) to the solution $\boldsymbol{u}$ of (1), we denote the errors in subdomain $\Omega_{1}$ by $\boldsymbol{d}^{n}:=\boldsymbol{u}-\boldsymbol{v}^{n}$ and in $\Omega_{2}$ by $\boldsymbol{e}^{n}:=\boldsymbol{u}-\boldsymbol{w}^{n}$. The error equations are 


$$
\begin{aligned}
\partial_{t} \boldsymbol{d}^{n+1}-\Delta \boldsymbol{d}^{n+1}+\boldsymbol{f}(\boldsymbol{u})-\boldsymbol{f}\left(\boldsymbol{v}^{n+1}\right) & =0 & & \text { in } \Omega_{1} \times(0, T), \\
\boldsymbol{d}^{\boldsymbol{n}+\mathbf{1}}(\beta L, t) & =\boldsymbol{e}^{n}(\beta L, t) & & \text { on }(0, T), \\
\partial_{t} \boldsymbol{e}^{n+1}-\Delta \boldsymbol{e}^{n+1}+\boldsymbol{f}(\boldsymbol{u})-\boldsymbol{f}\left(\boldsymbol{w}^{n+1}\right) & =0 & & \text { in } \Omega_{2} \times(0, T), \\
\boldsymbol{e}^{\boldsymbol{n}+\mathbf{1}}(\alpha L, t) & =\boldsymbol{d}^{n}(\alpha L, t) & & \text { on }(0, T),
\end{aligned}
$$

where the initial and boundary conditions on the exterior boundaries are zero, since the error vanishes there. Using a Taylor expansion with remainder term in Lagrange form, we obtain for $i=1,2$

$$
\begin{aligned}
& \partial_{t} d_{i}^{n+1}-\Delta d_{i}^{n+1}+\partial_{1} f_{i}\left(\xi_{i, 1}, u_{2}\right) d_{1}^{n+1}+\partial_{2} f_{i}\left(v_{1}^{n+1}, \xi_{i, 2}\right) d_{2}^{n+1}=0 \text { in } \Omega_{1} \times(0, T), \\
& \partial_{t} e_{i}^{n+1}-\Delta e_{i}^{n+1}+\partial_{1} f_{i}\left(\xi_{i, 1}^{\prime}, u_{2}\right) e_{1}^{n+1}+\partial_{2} f_{i}\left(w_{1}^{n+1}, \xi_{i, 2}^{\prime}\right) e_{2}^{n+1}=0 \text { in } \Omega_{2} \times(0, T),
\end{aligned}
$$

a linear system with variable coefficients, depending on the Jacobian of the non-linearity.

Our convergence analysis is based on upper solutions for the errors, which are constant in time.

Theorem 1 (Linear Convergence Estimate). Assume that $\partial_{1} f_{1} \geq 0$ and $\partial_{2} f_{2} \geq 0$, and that there exists a constant a satisfying $0<a<(\pi / L)^{2}$, such that $-a \leq \partial_{1} f_{2} \leq 0$ and $-a \leq \partial_{2} f_{1} \leq 0$. Then the errors in the Schwarz waveform relaxation algorithm (5) satisfy

$$
\begin{aligned}
& \sup _{x \in \Omega_{1}}\left\|\boldsymbol{d}^{2 n+1}(x, \cdot)\right\|_{\infty} \leq C_{1} \gamma^{k}\left\|\boldsymbol{e}^{0}(\beta L, \cdot)\right\|_{\infty}, \\
& \sup _{x \in \Omega_{2}}\left\|\boldsymbol{e}^{2 n+1}(x, \cdot)\right\|_{\infty} \leq C_{2} \gamma^{k}\left\|\boldsymbol{d}^{0}(\alpha L, \cdot)\right\|_{\infty},
\end{aligned}
$$

where $\gamma$ in $(0,1)$ is

$$
\gamma=\left(\frac{\sin (\sqrt{a} \alpha L)}{\sin (\sqrt{a} \beta L)}\right)\left(\frac{\sin (\sqrt{a}(1-\beta) L)}{\sin (\sqrt{a}(1-\alpha) L)}\right),
$$

and the constants $C_{1,2}$ are given by

$$
C_{1}=\sup _{0<x<\beta L} \frac{\sin (\sqrt{a} x)}{\sin (\sqrt{a} \beta L)}, \quad C_{2}=\sup _{\alpha L<x<L} \frac{\sin (\sqrt{a}(L-x))}{\sin (\sqrt{a}(1-\alpha) L)} .
$$

Proof. Let $M:=\max \left\{\left\|e_{1}^{n}(\beta L, \cdot)\right\|_{\infty},\left\|e_{2}^{n}(\beta L, \cdot)\right\|_{\infty}\right\}$ and define the function $\tilde{d}^{n+1}:=M \sin (\sqrt{a} x) / \sin (\sqrt{a} \beta L)$, which is the unique solution of the steady state problem

$$
-\Delta \tilde{d}^{n+1}-a \tilde{d}^{n+1}=0, \quad \text { with } \quad \tilde{d}^{n+1}(0)=0, \quad \tilde{d}^{n+1}(\beta L)=M .
$$

In order to show that $\tilde{d}^{n+1}$ is a supersolution of the two errors $d_{1}^{n+1}$ and $d_{2}^{n+1}$, we consider the differences $\hat{d}_{1}^{n+1}:=\tilde{d}^{n+1}-d_{1}^{n+1}$ and $\hat{d}_{2}^{n+1}:=\tilde{d}^{n+1}-d_{2}^{n+1}$, which satisfy in $\Omega_{1}$ the system of equations

$$
\begin{aligned}
& \partial_{t} \hat{d}_{1}^{n+1}-\Delta \hat{d}_{1}^{n+1}-a \tilde{d}^{n+1}-\partial_{1} f_{1}\left(\xi_{1,1}, u_{2}\right) d_{1}^{n+1}-\partial_{2} f_{1}\left(v_{1}^{n+1}, \xi_{1,2}\right) d_{2}^{n+1}=0 \\
& \partial_{t} \hat{d}_{2}^{n+1}-\Delta \hat{d}_{2}^{n+1}-a \tilde{d}^{n+1}-\partial_{1} f_{2}\left(\xi_{2,1}, u_{2}\right) d_{1}^{n+1}-\partial_{2} f_{2}\left(v_{1}^{n+1}, \xi_{2,2}\right) d_{2}^{n+1}=0 .
\end{aligned}
$$


Adding and subtracting $\partial_{1} f_{1}\left(\xi_{1,1}, u_{2}\right) \tilde{d}^{n+1}$ and $\partial_{2} f_{1}\left(v_{1}^{n+1}, \xi_{1,2}\right) \tilde{d}^{n+1}$ in the first equation, and $\partial_{1} f_{2}\left(\xi_{2,1}, u_{2}\right) \tilde{d}^{n+1}$ and $\partial_{2} f_{2}\left(v_{1}^{n+1}, \xi_{2,2}\right) \tilde{d}^{n+1}$ in the second equation, we obtain

$$
\begin{aligned}
\partial_{t} \hat{d}_{1}^{n+1}-\Delta \hat{d}_{1}^{n+1}= & -\partial_{1} f_{1}\left(\xi_{1,1}, u_{2}\right) \hat{d}_{1}^{n+1}-\partial_{2} f_{1}\left(v_{1}^{n+1}, \xi_{1,2}\right) \hat{d}_{2}^{n+1} \\
& +\partial_{1} f_{1}\left(\xi_{1,1}, u_{2}\right) \tilde{d}^{n+1}+\left(a+\partial_{2} f_{1}\left(v_{1}^{n+1}, \xi_{1,2}\right)\right) \tilde{d}^{n+1} \\
\partial_{t} \hat{d}_{2}^{n+1}-\Delta \hat{d}_{2}^{n+1}= & -\partial_{1} f_{2}\left(\xi_{2,1}, u_{2}\right) \hat{d}_{1}^{n+1}-\partial_{2} f_{2}\left(v_{1}^{n+1}, \xi_{2,2}\right) \hat{d}_{2}^{n+1} \\
& +\left(a+\partial_{1} f_{2}\left(\xi_{2,1}, u_{2}\right)\right) \tilde{d}^{n+1}+\partial_{2} f_{2}\left(v_{1}^{n+1}, \xi_{2,2}\right) \tilde{d}^{n+1} .
\end{aligned}
$$

Under the assumptions of the theorem, and using the fact that $\tilde{d}^{n+1}$ is strictly positive in the interior of subdomain $\Omega_{1}$, we obtain the system of inequalities

$$
\begin{aligned}
& \partial_{t} \hat{d}_{1}^{n+1}-\Delta \hat{d}_{1}^{n+1}+\partial_{1} f_{1}\left(\xi_{1,1}, u_{2}\right) \hat{d}_{1}^{n+1}+\partial_{2} f_{1}\left(v_{1}^{n+1}, \xi_{1,2}\right) \hat{d}_{2}^{n+1} \geq 0, \\
& \partial_{t} \hat{d}_{2}^{n+1}-\Delta \hat{d}_{2}^{n+1}+\partial_{1} f_{2}\left(\xi_{2,1}, u_{2}\right) \hat{d}_{1}^{n+1}+\partial_{2} f_{2}\left(v_{1}^{n+1}, \xi_{2,2}\right) \hat{d}_{2}^{n+1} \geq 0 .
\end{aligned}
$$

Since $\partial_{2} f_{1}\left(v_{1}^{n+1}, \xi_{1,2}\right) \leq 0$ and $\partial_{1} f_{2}\left(\xi_{2,1}, u_{2}\right) \leq 0$, we can now apply Lemma 2 to conclude that $\tilde{d}_{1}^{n+1}=\tilde{d}^{n+1}-d_{1}^{n+1} \geq 0$ and $\hat{d}_{2}^{n+1}=\tilde{d}^{n+1}-d_{2}^{n+1} \geq 0$ in $\Omega_{1}$. Using a similar argument for the sums, we obtain that also $\tilde{d}^{n+1}+d_{1}^{n+1} \geq 0$ and $\tilde{d}^{n+1}+d_{2}^{n+1} \geq 0$, which implies by the positivity of $\tilde{d}^{n+1}$ that their modulus is bounded, and we have for $i=1,2$

$$
\left|d_{i}^{n+1}(x, t)\right| \leq \tilde{d}^{n+1}=\max \left\{\left\|e_{1}^{n}(\beta L, \cdot)\right\|_{\infty},\left\|e_{2}^{n}(\beta L, \cdot)\right\|_{\infty}\right\} \frac{\sin (\sqrt{a} x)}{\sin (\sqrt{a} \beta L)} .
$$

Using a similar argument on subdomain $\Omega_{2}$, we obtain for $i=1,2$

$$
\left|e_{i}^{n+1}(x, t)\right| \leq \max \left\{\left\|d_{1}^{n}(\alpha L, \cdot)\right\|_{\infty},\left\|d_{2}^{n}(\alpha L, \cdot)\right\|_{\infty}\right\} \frac{\sin (\sqrt{a}(L-x))}{\sin (\sqrt{a}(1-\alpha) L)} .
$$

Since the bounds are uniform in $t$, we obtain over a double step

$$
\left\|\boldsymbol{d}^{n+1}(\alpha L, \cdot)\right\|_{\infty} \leq \gamma\left\|\boldsymbol{d}^{n-1}(\alpha L, \cdot)\right\|_{\infty}, \quad\left\|\boldsymbol{e}^{n+1}(\beta L, \cdot)\right\|_{\infty} \leq \gamma\left\|\boldsymbol{e}^{n-1}(\beta L, \cdot)\right\|_{\infty},
$$

and by induction (7) and (8). The fact that $\gamma<1$ has been shown already in [2].

\section{Numerical Results}

We present numerical results for three different semilinear systems: the Belousov-Zhabotinsky equations, the FitzHugh-Nagumo equations, and the Lotka-Volterra system with migration. All numerical experiments are performed in the domain $\Omega=(0,1)$ and on the time interval $(0, T)$, with $T=12 \pi$. We discretize the equations with a standard three point finite difference method in space (with mesh size $\Delta x=\frac{1}{20}$ ), and a semi-implicit Euler time-discretization scheme (with time step $\Delta t=\frac{\pi}{10}$ ), where implicit integration is used for the diffusive term and explicit integration for the reaction term. The space-time domain $\Omega$ is decomposed into two overlapping domains (with overlap size $\delta=2 \Delta x$ ) and we use Dirichlet conditions at the interfaces. 


\subsection{Belousov-Zhabotinsky Equations}

The Belousov-Zhabotinsky equations model non-equilibrium thermodynamics, resulting in the establishment of a nonlinear chemical oscillator, see [9], page 322 for details. They are given by

$$
\begin{aligned}
& \partial_{t} u_{1}-\frac{1}{2} \partial_{x x} u_{1}-u_{1}\left(1-u_{1}-r u_{2}\right)=0, \\
& \partial_{t} u_{2}-\frac{1}{2} \partial_{x x} v+b u_{1} u_{2}=0 .
\end{aligned}
$$

The hypotheses of Theorem 1 are satisfied for this system after performing the change of variables $\tilde{u}_{1}=1-u_{1}, \tilde{u}_{2}=u_{2}$, under the condition that the components $u_{1}$ and $u_{2}$ remain positive. This condition holds, provided that the initial conditions satisfy $u_{1}(x, 0)=u_{1,0}(x) \geq 0$ and $u_{2}(x, 0)=u_{2,0}(x) \geq 0$. Figure 2 shows the linear convergence predicted by the convergence bound of Theorem 1.

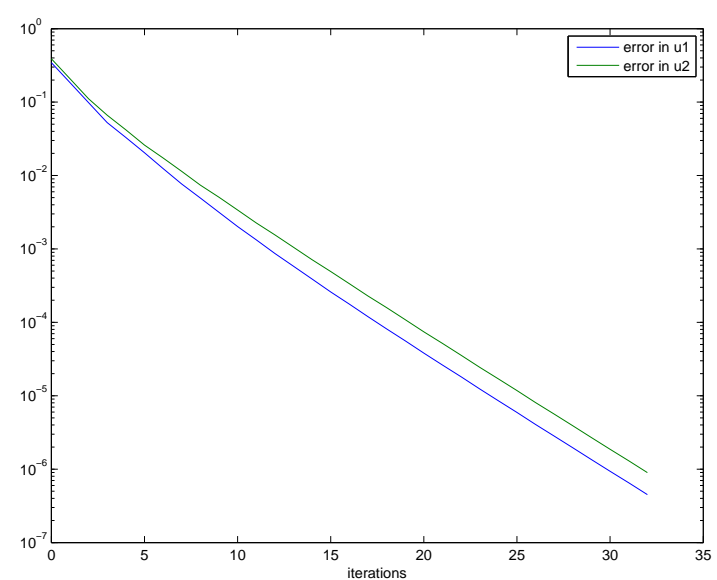

Fig. 2. Convergence history for the Belousov-Zhabotinsky equations

\subsection{FitzHugh-Nagumo Equations}

The system of reaction diffusion equations

$$
\begin{aligned}
& \partial_{t} u_{1}-\frac{1}{2} \partial_{x x} u_{1}-f\left(u_{1}\right)+u_{2}=0, \\
& \partial_{t} u_{2}-\frac{1}{2} \partial_{x x} u_{2}-u_{1}+u_{2}=0,
\end{aligned}
$$

with $f\left(u_{1}\right)=u_{1}-u_{1}^{3}$ is called the FitzHugh-Nagumo equations, and describes how an action potential travels through a nerve. It is the prototype of an excitable system (e.g., a neuron) or an activator-inhibitor system: close to the ground state, one component stimulates the production of both components, while the other one inhibits their growth, see [9], page 161 for details. This system does not satisfy the hypotheses of Theorem 1, but nevertheless we observe linear convergence, as shown in Figure 3. 


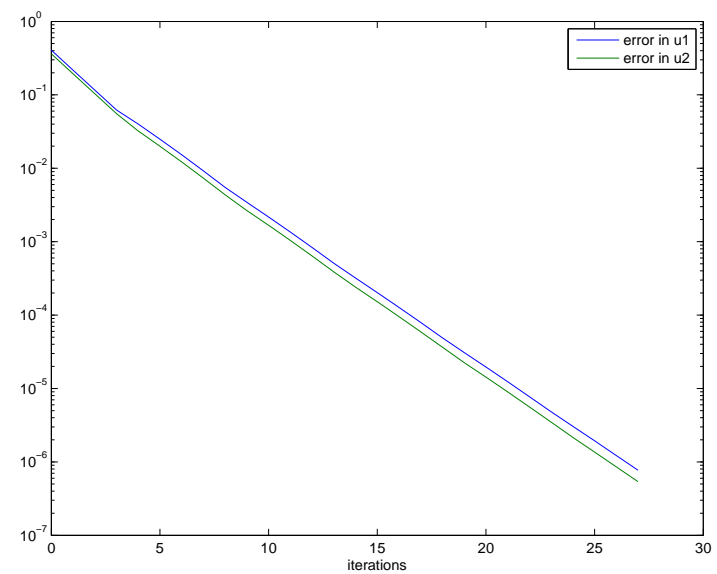

Fig. 3. Convergence history for the FitzHugh-Nagumo equations

\subsection{Lotka-Volterra Equations}

The Lotka-Volterra equations with migration term are

$$
\begin{aligned}
& \partial_{t} u_{1}-\frac{2}{25} \partial_{x x} u_{1}-u_{1}\left(1-u_{2}\right)=0, \\
& \partial_{t} u_{2}-\frac{2}{25} \partial_{x x} u_{2}+u_{2}\left(1-u_{1}\right)=0,
\end{aligned}
$$

and they describe a biological predator-prey system, where both predator and prey are migrating randomly. This system does not satisfy the hypotheses of Theorem 1, and now we observe quite a different convergence behavior, as shown in Figure 4.

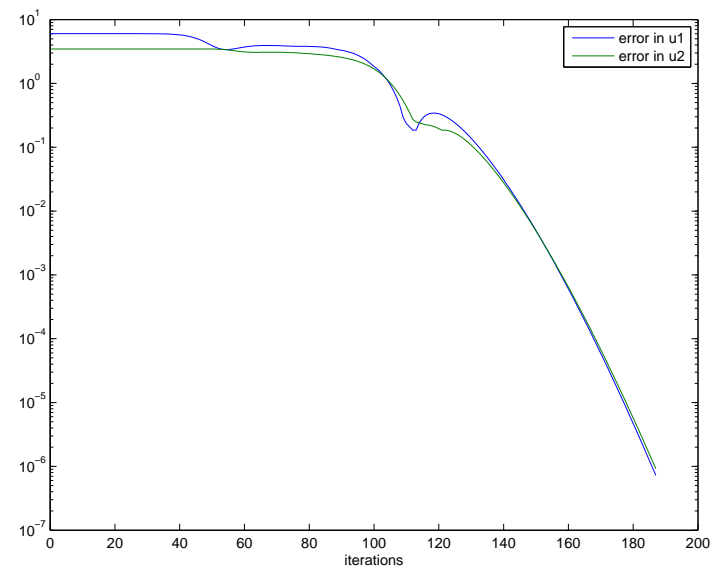

Fig. 4. Convergence history for the Lotka-Volterra equations with migration 


\section{Conclusions}

Schwarz waveform relaxation algorithms often exhibit superlinear convergence, as observed in the last example, see for example [2]. A corresponding convergence analysis requires however quite different techniques from the ones we have presented here, and will appear in an upcoming paper.

\section{References}

[1] Martin J. Gander. Overlapping Schwarz waveform relaxation for parabolic problems. In J. Mandel, C. Farhat, and X.-C. Cai, editors, Tenth International Conference on Domain Decomposition Methods. AMS, Contemporary Mathematics 218, 1998.

[2] Martin J. Gander. A waveform relaxation algorithm with overlapping splitting for reaction diffusion equations. Numerical Linear Algebra with Applications, 6:125-145, 1998.

[3] Martin J. Gander, Laurence Halpern, and Frédéric Nataf. Optimal convergence for overlapping and non-overlapping Schwarz waveform relaxation. In C-H. Lai, P. Bjørstad, M. Cross, and O. Widlund, editors, Eleventh international Conference of Domain Decomposition Methods. ddm.org, 1999.

[4] Martin J. Gander, Laurence Halpern, and Frédéric Nataf. Optimal Schwarz waveform relaxation for the one dimensional wave equation. SIAM Journal of Numerical Analysis, 41(5):1643-1681, 2003.

[5] Martin J. Gander and Christian Rohde. Overlapping Schwarz waveform relaxation for convection dominated nonlinear conservation laws. SIAM J. Sci. Comp., 27(2):415-439, 2005.

[6] Martin J. Gander and Andrew M. Stuart. Space time continuous analysis of waveform relaxation for the heat equation. SIAM J. Sci. Comp., 19:2014-2031, 1998.

[7] Eldar Giladi and Herbert B. Keller. Space time domain decomposition for parabolic problems. Numerische Mathematik, 93(2):279-313, 2002.

[8] Daniel Henry. Geometric theory of semilinear parabolic equations, volume 840 of Lecture Notes in Mathematics. Springer-Verlag, Berlin, 1981.

[9] J. D. Murray. Mathematical biology, volume 19 of Biomathematics. Springer-Verlag, Berlin, 1989.

[10] C. V. Pao. Nonlinear parabolic and elliptic equations. Plenum Press, New York, 1992.

[11] Aizik I. Volpert, Vitaly A. Volpert, and Vladimir A. Volpert. Traveling wave solutions of parabolic systems, volume 140 of Translations of Mathematical Monographs. American Mathematical Society, Providence, RI, 1994. Translated from the Russian manuscript by James F. Heyda. 